\title{
An Empirical Examination of Behavioral Factors in Creative Development of Game Prototypes
}

\author{
Michail N. Giannakos ${ }^{1}$, Letizia Jaccheri ${ }^{1}$, and Sandro Morasca ${ }^{2}$ \\ ${ }^{1}$ Norwegian University of Science and Technology, Trondheim, Norway \\ \{michailg, letizia\} @idi.ntnu.no \\ ${ }^{2}$ Università degli Studi dell'Insubria, Como, Italy \\ sandro.morasca@uninsubria.it
}

\begin{abstract}
In the last few years, several learning programs, workshops, technologies, and activities have been introduced and applied to game prototyping activities. The research goal of this work is to investigate how participants experience game prototyping activities. This paper presents a one-day intensive course consisting of both learning and hands-on sessions with open source software (OSS) tools, tangible materials, and sensor boards for creative development of games prototypes. The intensive course program was developed using the empirical experience of the instructors from numerous prior programs. We present the results of an empirical examination regarding participants' attitude towards the program. A group of $12 \mathrm{MSc} / \mathrm{PhD}$ students, teachers, and designers participated in the program in our empirical evaluation. We used a survey grounded in motivational factors for technology and open-ended questions to obtain both quantitative and qualitative data from the participants. Quantitative statistical analysis indicates that, in our study, participants' satisfaction and activity's usefulness are the most influential factors for participants' intention to attend similar activities in the future. Qualitative analysis suggests improvements on how to prepare the participants, introduce the software used in the courses, and enrich the variety of the materials in our program.
\end{abstract}

Keywords: Game Prototyping, Empirical Examination, Creative Development, Software Engineering Activities, Behavioral Factors, OSS for Entertainment.

\section{Introduction}

Computer games are highly engaging not only for game players but also for game developers. Here we mention The Gathering (http://www.gathering.org) as an example of a highly engaging environment for game players and developers. Thousands of youths have been meeting every year since 1992 at The Gathering, the second largest computer party in the world (second only to DreamHack). Events like The Gathering are a place for young creative people to engage in creative game development, in addiction to demo coding, music, graphics, animation, and game playing. These events receive a lot of attention by researchers and educators who aim at understanding this growing phenomenon of creative development of computer games. 
Modding, the practice and process of developing game mods, is typically a "Do It Yourself" (DIY) approach to game development [6] by reusing game software. For this purpose, well known and validated Software Engineering (SE) theories and techniques for development and innovation can be applied to game development. But little is known about how participants experience game prototyping activities. How easy it is for them to participate? Do they enjoy their participation to these activities? Do they intend to participate to future versions of such game prototyping activities?

In our approach, we developed and taught an intensive course for game prototyping and development and empirically evaluated it via the participants' feedback. Specifically, we employed a mixed (both quantitative and qualitative) approach to gain insights into participants' attitudes towards the intensive course, to examine any potential relations to the participants' intention to re-attend similar events in the future. In our work, we give insights regarding:

- Behavioral factors about game prototyping activities.

- Potential improvements for the development of games prototyping courses.

The clarification of participants' attitudes during these activities is expected to also shed a light on improvements on the course structure and development. We found that by increasing participants' satisfaction and connecting the activities with something more important (increase the activities' usefulness) allows instructors to increase participants' willingness to attend such intensive programs. In addition, participants complete the intensive course more successfully if they are provided with more information prior to the intensive course. In our case, such information included the OSS, sensor platform, and materials used.

\section{Related Work}

The previous decades have seen various attempts to introduce creative learning practices in CS and SE. Numerous projects focus on creativity and design using diverse software tools (e.g., VVVV, PD, Scratch, Processing, Panda3D, Alice, Wiring). Most of these projects build on the context of robotics, interactive installations, and creativity. The role of the active learner and project based learning with multidisciplinary teams in CS and SE have been explored in the literature [3] [6] [7] with great success. For example, one or more stakeholders to a project may have different backgrounds than the rest of a team, or when the given task is to "think outside the box" by developing creative solutions.

Games prototyping and development courses engage students through incorporating up-to-date technologies, providing the opportunity to work on projects that are relevant to them, and instructing students on the ethics and methods of research. Our activity can be characterized as a project-based learning activity and emphasizes in the challenging balance between production and process [1]. Related studies [6] [7] have proved the value of game prototyping in SE. While these studies address the issue of creative development of computer games, they do not explicitly explore how these activities are experienced by participants and can be potentially improved. 


\section{$3 \quad$ Methodology}

\subsection{The Intensive Course}

A full-day intensive course was organized as a combination of a learning session and hands-on applied sessions with OSS tools for creativity [4]. The learning sessions derived from both related literature and the research experience [2] [5] of the two instructors (who are also the first two authors of this paper). The hands-on sessions had the goal of letting the participants experiment with software tools for creative game development while working in small groups to get to know each other in a creative and motivating atmosphere. The first half of the course included presentations on the background of the topics. The second half of the course included hands-on creative sessions. The participants worked in teams on small creative tasks using the methods presented earlier. The exact schedule of the intensive course can be found here: http://wp.me/PXD0L-tm. After the course we proceeded with the evaluation. Due to the schedule of the intensive course and the need to have a low ratio among instructors and participants, our evaluation was not a large scale one. To fill this gap, we used both qualitative and quantitative data to interpret and summarize our results.

\subsection{Participants and Procedures}

Twelve participants attended the one full-day intensive course. The participants were $\mathrm{MSc} / \mathrm{PhD}$ students in Software Engineering, software designers, and software teachers. The participants formed four different teams. Each team made a character (e.g., Mr. Coffee in Figure 1) using physical materials (e.g., Lego objects, plastic, glass). They then imported the character in Scratch, developed a storyboard for the game, and programmed the game on Scratch. At the end of the course, four different projects had been developed based on the given theme, which concerned issues with the Earth's water systems. In Figure 1, we present an example storyboard and the corresponding game developed by one of the teams.

\begin{tabular}{|l|l|}
$\begin{array}{l}\text { Storyboard } \\
\text { Sam is really tired! He needs a cup of coffee to wake up and go to work! } \\
\text { Unfortunately, there is not enough water in the tap. Water flows from } \\
\text { ponds which are filled by rain and dried by the sun. } \\
\text { Mr. Coffee is Sam's best friend! He tries to help Sam get water for his } \\
\text { coffee machine in the following way: } \\
\text { 1. Water flows from several ponds through pipes to Sam's coffee machine } \\
\text { 2. If the sun shines on a pond it becomes dry } \\
\text { 3. If the cloud rains into a pond it gets filled } \\
\text { 4. Mr. Coffee carries an umbrella } \\
\text { 5. Mr. Coffee can cover a pond with his umbrella to prevent it from drying } \\
\text { up } \\
\text { 6. Once there is enough water in Sam's machine a button starts making } \\
\text { coffee } \\
\text { a) Controlling Mr. Coffee The slider moves Mr. Coffee sideways. } \\
\text { b) By pressing the button he either takes or drops the umbrella. }\end{array}$ \\
\hline
\end{tabular}

Fig. 1. One team developed a Scratch game with the name Mr. Coffee, the interface of the game (left) and the Storyboard (right) 


\subsection{Measures}

The research methodology included a survey composed of three parts: the first included questions on the demographics of the participants (occupation and age); the second part included measures of the various factors identified in the literature from previous research; and the third consisted of open-entry questions regarding the recommendations and feedback of the participants. The questionnaire factors were: a) Satisfaction-the degree to which the participant positively feels about the course, b) Intention to Re-attend-the degree of participant's willingness to re-attend the course, c) Easiness-how easy it is for participants to shop online, d) Enjoyment-the degree to which the course is perceived to be personally enjoyable, e) Usefulness-the degree to which the participant believes that attending the course is useful, f) Control-the degree to which a participant perceives how easy or difficult it would be to perform an operation in the course, g) Happiness-the degree to which a person felt happy during the activity, h) Anxiety-the degree to which the participant felt anxious during the course. In all cases, 7-point Likert scales were used to measure the variables. The participants completed the questionnaire at the end of the course.

\section{$4 \quad$ Data Analysis and Results}

We carried out quantitative and qualitative analyses on the data obtained from the survey. We first carried out a quantitative analysis, which was based on the factors measured in the questionnaire (Table 1). We proceed to test the reliability of each measure using Cronbach $\alpha$ coefficient. As Table 1 shows, the result of the test revealed acceptable indices of reliability in all the factors $(>0.7)$. We also computed descriptive statistics for the factors under investigation. The factors is found to be in satisfactory level; however, participants' intention to re-attend in similar course is not as high as we would like (see Table 1 for the exact values).

Table 1. Summary of measurement scales

\begin{tabular}{|l|c|c|c|c|c|c|c|c|c|c|c|}
\hline Factors & Mean & SD & CR & STF & IRa & EAS & ENJ & USF & CON & HAP & ANX \\
\hline $\begin{array}{l}\text { Satisfaction } \\
\text { (STF) }\end{array}$ & 5.81 & 0.78 & 0.87 & 1.0 & & & & & & & \\
\hline $\begin{array}{l}\text { Intention to Re- } \\
\text { attend (IRa) }\end{array}$ & 4.69 & 1.46 & 0.95 & $\mathbf{. 7 8 * *}$ & 1.0 & & & & & & \\
\hline Easiness (EAS) & 5.48 & 0.94 & 0.74 & -.17 & -.16 & 1.0 & & & & & \\
\hline Enjoyment (ENJ) & 5.77 & 0.82 & 0.85 & .54 & .46 & -.19 & 1.0 & & & & \\
\hline Usefulness (USF) & 5.04 & 1.26 & 0.87 & .52 & $\mathbf{. 6 0}$ & .31 & .20 & 1.0 & & & \\
\hline Control (CON) & 6.04 & 0.58 & 0.85 & .49 & .13 & .27 & $\mathbf{. 5 8}$ & .16 & 1.0 & & \\
\hline Happiness (HAP) & 5.58 & 0.81 & 0.79 & .45 & .43 & .23 & $\mathbf{. 6 3}^{*}$ & .02 & $\mathbf{. 6 7}$ & 1.0 & \\
\hline Anxiety (ANX) & 2.69 & 0.98 & 0.83 & -.25 & -.28 & -.24 & $\mathbf{- . 5 8}$ & .11 & -.51 & $\mathbf{. . 8 2}$ & 1.0 \\
\hline
\end{tabular}

SD, Standard Deviation; CR, Cronbach $\alpha$; Correlation is significant at the ** $0.01 \& * 0.05$ level. 
To investigate any possible relationships among the factors and explore factors that may be strongly related to participants' intention to re-attend the course, we used the Spearman's rank-correlation coefficient, statistically testing the null hypothesis which is equal to 0. Spearman's test suggests that some of the factors are related, in some cases relatively strongly. More precisely, the most important positive relationships involve intention to re-attend on the one side and satisfaction and usefulness on the other side. In addition, enjoyment is positively related to control and to happiness, and control is positively related to happiness. These relations revealed the important role of control over the course and its high influence on the participants' positive feelings during the course. Moreover, there is also one expected negative relation, that of happiness with anxiety. We present the results of Spearman's test in Table 1.

In addition to the above questions, we used free-entry questions to capture any additional information and suggestions from the participants. The responses from freeentry questions and the observations of the instructors during the course form the qualitative part of our study. After collecting the responses, we proceeded with a content analysis. Based on the free text responses, we summarize the following additions that will potentially improve the course:

1. It was difficult for the participants to start working with a software that was new to them: sending information regarding the software prior to the course will familiarize participants with the software, thereby making the intensive course more effective;

2. Longer, in-depth introduction of the software at the beginning of the course will also help the participants be more effective in the second (hands-on) part of the course;

3. The physical part was very important, and supplying participants with a wider range of physical materials may allow them to more quickly develop a more representative physical character.

\section{Conclusions}

In the course, the twelve participants were introduced to OSS practices and tools that are suitable for creators of interactive and playful systems. The course offered the participants an opportunity to create their physical character through OSS tools, such as Scratch and Pico-Boards, which motivate a broader participation of technical and non-technical users in the creative production of interactive systems.

The first step was to design and deploy a series of these activities. In particular, participants engaged in OSS Scratch and the hardware platform Pico-board, which enable them to engage in the world of creativity with digital games and stories. In the final step, we used the empirical data obtained from participants' experiences with the course to evaluate it and investigate any improvements.

This study, based on the quantitative analyses, particularly improves our understandings regarding creative development of games prototypes. The findings indicate that satisfaction and usefulness have positive relationships with participants' intention to participate in similar activities in the future. As such, by increasing their 
satisfaction and connecting the activity to something more important (increase activity's usefulness), we will be able to increase participants' willingness to attend such as intensive programs.

The qualitative analyses suggest guidelines regarding how to prepare the participants, how to introduce the OSS, and how to enrich the variety of the materials in our program. Specifically, educating participants about the software prior to the intensive course and conducting an in-depth introduction at the beginning will help them "buy time" from the intensive course, allowing for further progress. Moreover, as the cornerstone of our program is creativity, the supply of a wider range of physical materials will allow students to produce a physical object that is more advanced and closer to what they want, in a shorter amount of time.

As with most of the empirical studies, there are a few limitations. As for the internal validity of our study, the data are based on a self-reported method and instructors' observations. Other in-depth methods such as semi-structured interviews could provide a complementary picture of the findings through data triangulation. As for external validity, the subjects were $\mathrm{MSc} / \mathrm{PhD}$ students, teachers and designers, which may somewhat limit the generalizability of our results. Nevertheless, the insights drawn are not connected with the subjects' background and can be applied on any population. Another direction of research is to apply the empirical framework proposed in this paper to the investigation of existing highly engaging environment for game players and developers, such as The Gathering.

Acknowledgements. We are grateful to the participants of the course and to K. Chorianopoulos and A.S. Nossum for their help on the prior versions of this program.

\section{References}

1. Chaffin, A., Barnes, T.: Lessons from a course on serious games research and prototyping. In: Proceedings of the Fifth International Conference on the Foundations of Digital Games (FDG 2010), pp. 32-39. ACM, NY (2010)

2. Chorianopoulos, K., Jaccheri, L., Nossum, A.S.: Creative and open software engineering practices and tools in maker community projects. In: Proceedings of the 4th ACM SIGCHI Symposium on Engineering Interactive Computing Systems, pp. 333-334. ACM (2012)

3. Holbert, K.E., Karady, G.G.: Strategies, challenges and prospects for active learning in the computer-based classroom. IEEE Trans. Educ. 52(1), 31-38 (2009)

4. Jaccheri, L., Giannakos, M.N.: Open Source Software for Entertainment. In: Herrlich, M., Malaka, R., Masuch, M. (eds.) ICEC 2012. LNCS, vol. 7522, pp. 604-607. Springer, Heidelberg (2012)

5. Jaccheri, L.: Open software and art: a tutorial. In: Anacleto, J.C., Fels, S., Graham, N., Kapralos, B., Saif El-Nasr, M., Stanley, K. (eds.) ICEC 2011. LNCS, vol. 6972, pp. 468-471. Springer, Heidelberg (2011)

6. Scacchi, W.: Modding as a basis for developing game systems. In: Proceedings of GAS 2011, pp. 5-8. ACM, NY (2011)

7. Wang, A.I.: Extensive Evaluation of Using a Game Project in a Software Architecture Course. Trans. Comput. Educ. 11(1), Article 5, 28 pages (2011), doi:1921607.1921612 